\title{
GAS-LIQUID INTERFACIAL AREA IN THE OXYGEN ABSORPTION TO OIL-IN-WATER EMULSIONS IN AN AIRLIFT REACTOR
}

\author{
D. Gómez-Díaz, ${ }^{1 *}$ N. Gomes, ${ }^{2}$ J. A. Teixeira ${ }^{2}$ and I. Belo ${ }^{2}$ \\ 1. Department of Chemical Engineering, University of Santiago de Compostela, Campus Sur, Santiago de Compostela E-15782, \\ Spain \\ 2. IBB-Institute for Biotechnology and Bioengineering, Center of Biological Engineering, University of Minho, Campus de \\ Gualtar, Braga 4710-057, Portugal
}

\begin{abstract}
The present work includes an exhaustive study about gas-liquid interfacial area between gas phase and liquid heterogeneous medium generated in an airlift bioreactor. The system studied is composed by water, methyl ricinoleate (MR), and Tween-80, since it is the base of the medium used for the production of $\gamma$-decalactone, a peach-like aroma compound of industrial interest that can be produced biotechnologically through the biotransformation of ricinoleic acid, by the yeast Yarrowia lipolytica. Experimental results allow describing the hydrodynamic behaviour of the gas phase into the biphasic medium.

Le travail actuel comprend une étude approfondie sur la zone interfaciale gaz-liquide entre la phase gazeuse et le milieu hétérogène liquide produit par un bioréacteur à agitation par circulation d'air. Le système étudié se compose d'eau, de ricinoléate de méthyle et de Tween 80 , puisqu'il constitue la base du milieu utilisé pour la production de $\gamma$-decalactone, un composé d'arôme semblable à la pêche d'intérêt industriel que l'on peut produire de façon biotechnologique par la biotransformation de l'acide ricinoléique (un important constituant du ricinoléate de méthyle), par la levure Yarrowia lipolytica. Les résultats expérimentaux permettent de décrire le comportement hydrodynamique de la phase gazeuse dans le milieu biphasique.
\end{abstract}

Keywords: airlift bioreactor, methyl ricinoleate, emulsion, gas-liquid interfacial area

\section{INTRODUCTION}

$\mathrm{G}$ as-liquid-liquid systems are encountered in many reactions and have gained special interest in chemical engineering with the introduction of homogeneous biphasic catalysis. Mass transfer modelling in biphasic media has been done by several authors using as a model, systems in which the second liquid phase (organic) is an additional inert and external compound (like perfluorocarbons and silicone oils, among others) added to the system on the purpose to improve mass transfer from the gas to the liquid aqueous phase (Dumont and Delmas, 2003). These systems are encountered in some biochemical applications where a hydrophobic compound with higher oxygen solubility than water is used to improve the oxygen transfer rate to the culture media. However, numerous biotechnological processes are based on the development of microorganisms within a biphasic medium formed by an oil-in-water emulsion where the oil is the substrate to be degraded. In these cases, the organic substrate can retard the gas-liquid mass transfer, thus limiting the overall productivity.

The system herein presented is an emulsion of water and methyl ricinoleate (MR), stabilised by Tween- 80 , which constitutes the medium used in the production of $\gamma$-decalactone (a peach-like aroma compound of industrial interest) through the biotransformation of ricinoleic acid (major constituent of MR) by the yeast Yarrowia lipolytica (Aguedo et al., 2005). The biochemical

\footnotetext{
* Author to whom correspondence may be addressed.

E-mail address: diego.gomez@usc.es

Can. J. Chem. Eng. 88:561-564, 2010.

(C) 2010 Canadian Society for Chemical Engineering DOI 10.1002/cjce.20307

Published online 5 May 2010 in Wiley InterScience

(www.interscience.wiley.com)
} 
Table 1. Experimental conditions employed in the present work

\begin{tabular}{lc}
\hline Variable & Range \\
\hline MR concentration & $0-0.54 \%(\mathrm{v} / \mathrm{v})$ \\
Tween-80 concentration & $0-0.093 \%(\mathrm{v} / \mathrm{v})$ \\
Gas flow-rate & $0.25-1.35 \mathrm{Lmin}^{-1}$ \\
\hline
\end{tabular}

pathway leading to the production of the aroma has been extensively studied. However, crucial steps controlling the production ratio of $\gamma$-decalactone against derived non-aromatic lactones are not totally understood. The oxygen is believed to influence these side-reactions, but this point remains to elucidate. Since Y. lipolytica is an obligate aerobe, oxygen is a crucial operational parameter for the control of processes involving this yeast. Previous studies (Gomes et al., 2007) have analysed the influence of operating conditions on oxygen mass transfer and on $\gamma$-decalactone production in a bubbling stirred bioreactor. In the present work, an airlift bioreactor was used and the main aim of the study was to investigate the gas-liquid interfacial area in the previously mentioned biphasic medium in an airlift contactor, especially the influence of operating conditions and emulsion composition.

\section{EXPERIMENTAL DATA}

\section{Liquid Velocity}

The airlift contactor used in the present work was constructed in Perspex with $0.07 \mathrm{~m}$ inside diameter. The height of the riser-tube was $0.37 \mathrm{~m}$ with an inside diameter of $0.032 \mathrm{~m}$. Air was used as gas stream in the gas-liquid contactor and it was fed at the bottom of the bubble column using a five holes sparger.

The liquid phase employed in the present work was an emulsion formed by water and MR. Tween-80 was used to stabilise the emulsion. Table 1 resumes the experimental conditions analysed in the present study.

The velocity of liquid circulation was determined with the neutral buoyancy flow follower technique. A small square piece of plastic (side length, $0.005 \mathrm{~m}$; specific gravity, $1.03 \mathrm{~g} \mathrm{~cm}^{-3}$ ) was used as a flow follower. The time taken by the follower to traverse a known vertical distance $(0.2 \mathrm{~m})$ in the downcomer was noted and the flow velocity was calculated from an average of 10 measurements. The density of the follower was very close to that of the liquid. Figure 1 shows that the increase of the air flow rate

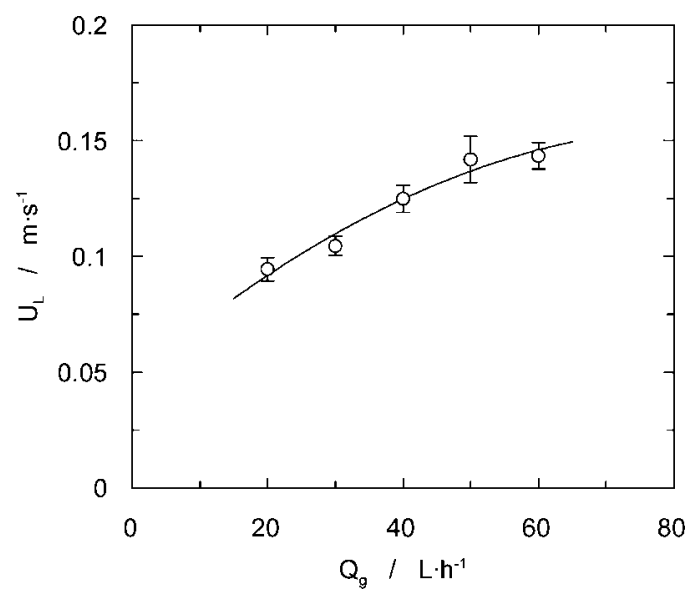

Figure 1. Liquid velocity $\left(U_{\mathrm{L}}\right)$ generated into the airlift reactor at different aeration rates. (up to certain limits) leads to an improvement in the liquid velocity, although it seems to be a trend to become constant at the higher aeration rates tested.

\section{Gas-Liquid Interfacial Area}

To determine the gas-liquid interfacial area, a photographic method was used. A bubble column, with geometrical characteristics of $4 \mathrm{~cm}$ inside side-length and height of $65 \mathrm{~cm}$, was used. Since the diameter and height are similar to those of the riser's airlift reactor, results can be extrapolated. The bubbles diameter was measured using a photographic method based on images of the bubbles taken along the height of the column, from the bottom to the top. The column height was divided in three sections and a minimum number of 50 well-defined bubbles along the column were used to evaluate the size distribution of the bubbles in the liquid phase for the different gas flow-rates tested.

The geometrical characteristics of the air bubbles produced in the contactor enable to calculate the value of the gas-liquid interfacial area. The bubbles produced in the contactor have ellipsoidal shape and for this reason, major $(E)$ and minor $(e)$ axes of the projected ellipsoid (in two dimensions) must be determined. The diameter of the equivalent sphere was taken as the representative bubble dimension. Equation (1) allows determining the diameter distribution of a bubble present along the gas-liquid contactor.

$d=\sqrt[3]{E^{2} e}$

Photographs in Figure 2 clearly indicate that an increase of the MR concentration in the liquid phase produces an increase in the bubbles size. The bubble diameter distribution along the bubble contactor has a direct influence upon the value corresponding to the gas-liquid interfacial area. The interfacial area determination is developed on the basis of two important parameters: the Sauter mean diameter and the gas hold-up. An example of the bubble size distribution obtained in the present work is shown in Figure 3. An increase of the surfactant concentration decreases the bubbles size along all the diameter range. This behaviour has an important role in the value of the gas-liquid interfacial area. The decrease of the liquid phase surface tension value, caused by the presence of surfactant molecules at gas-liquid interface, produces a decrease in the diameter of the bubbles generated into the gas-liquid contactor.

Different authors recommend the use of the Sauter mean diameter $\left(d_{32}\right)$ (Shah et al., 1982), which can be determined using the data calculated for the equivalent diameter by Equation (2):

$d_{32}=\frac{\sum_{i}\left(n_{i} d_{i}^{3}\right)}{\sum_{i}\left(n_{i} d_{i}^{2}\right)}$

where $n_{i}$ is the number of bubbles that have an equivalent diameter $\left(d_{i}\right)$.

The gas hold-up was determined in a square bubble column contactor, due to the difficulty to measure the variations on the height of the liquid in the airlift reactor. The use of this methodology is possible due to the absence of bubbles in the downcomer of the airlift reactor.

The gas hold-up, $\varepsilon_{\mathrm{G}}$, was calculated using the difference between the liquid height in the absence of gas in the bubble column, at different gas flow-rates (Equation 3) (Vasconcelos et 

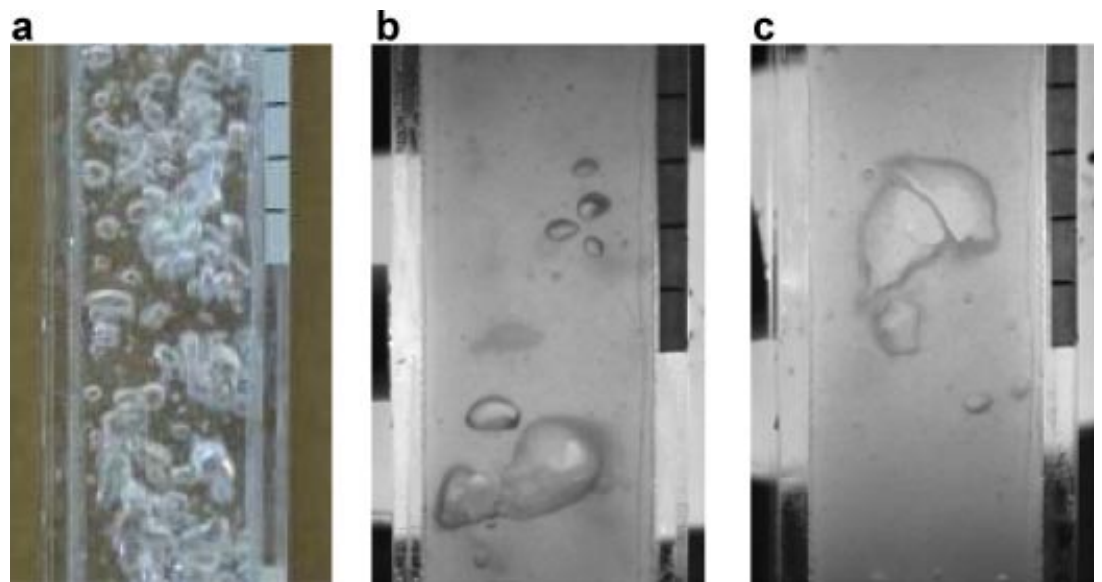

Figure 2. Photographs from the bubble column at an air flow-rate of $1.35 \mathrm{~L} \mathrm{~min}^{-1}$ for different liquid phase compositions: (a) aqueous solution with 0.093\% (v/v) Tween-80; (b) emulsion with 0.3\% (v/v) MR and 0.093\% (v/v) Tween-80; and (c) emulsion with 0.54\% (v/v) MR and 0.093\% (v/v) Tween-80.

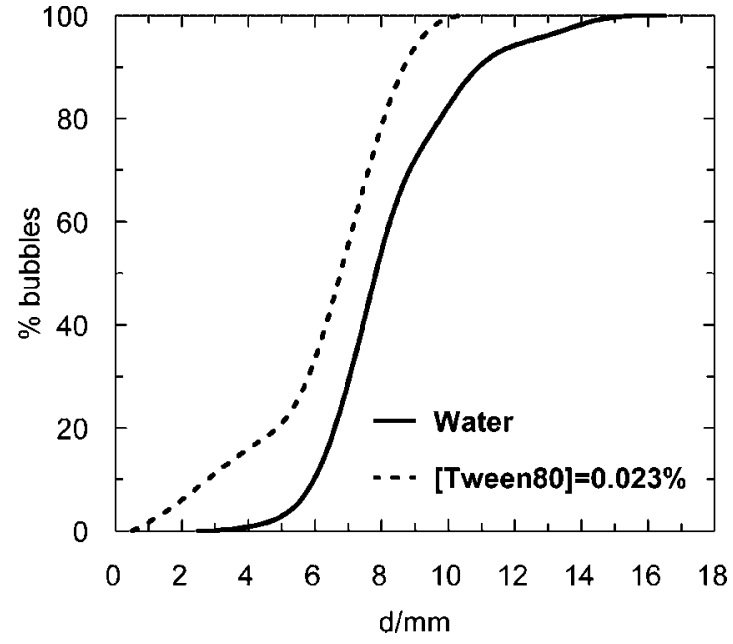

Figure 3. Influence of Tween-80 concentration in the liquid phase upon the bubble size. $Q_{G}=0.5 \mathrm{~L} \mathrm{~min}^{-1}$.

al., 2003):

$\varepsilon_{\mathrm{G}}=\frac{\Delta V}{\Delta V+V_{\mathrm{L}}}$

where $V_{\mathrm{L}}$ is the ungassed liquid volume and $\Delta V$ is the volume expansion after gas dispersion, calculated from the liquid level change and the cross sectional area. The variation of volume in the bubble column was calculated based on the difference observed in the liquid level before and after gassing.

The experimental results obtained for the gas hold-up in the bubble column are depicted in Figure 4 . An increase in the gas flow-rate (or surface gas velocity) produces a clear increase in the gas hold-up value with a linear trend. It can also be observed that there are no significant differences between the different liquid phase compositions tested, although for higher gas flow rates it seems that the gas hold-up becomes greater for the aqueous medium. In fact, since there is a bubbles size increase in water and therefore, its residence time decreases.

The Sauter mean diameter and the gas hold-up values allow the calculation of the specific gas-liquid interfacial area using

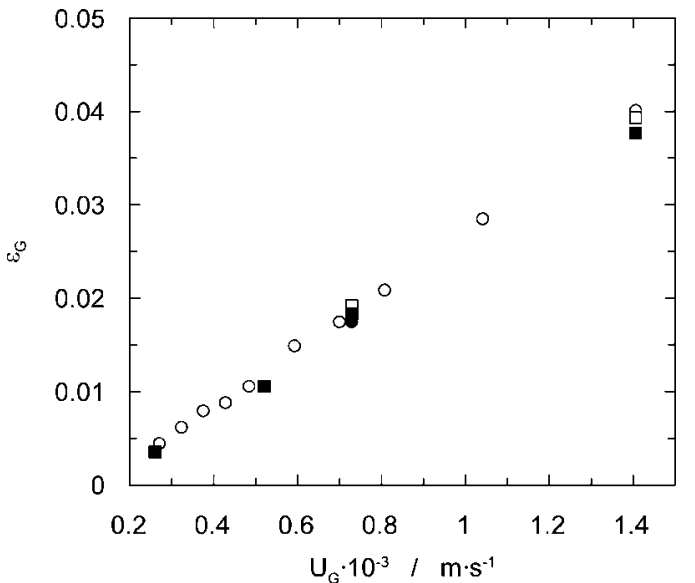

Figure 4. Influence of surface gas velocity $\left(U_{\mathrm{G}}\right)$ upon the gas hold-up $\left(\varepsilon_{\mathrm{G}}\right)$. (०) Pure water; $(\bullet) 0.093 \%(\mathrm{v} / \mathrm{v})$ Tween-80; $(\square) 0.1 \%(\mathrm{v} / \mathrm{v}) \mathrm{MR}$ and $0.093 \%(\mathrm{v} / \mathrm{v})$ Tween-80; ( $\mathbf{\square}) 0.54 \%(\mathrm{v} / \mathrm{v})$ MR and $0.093 \%(\mathrm{v} / \mathrm{v})$ Tween-80.

Equation (4) (van’t Riet and Tramper, 1991):

$a=\frac{6 \varepsilon_{\mathrm{G}}}{d_{32}\left(1-\varepsilon_{\mathrm{G}}\right)}$

This experimental methodology allows the determination of gas-liquid interfacial area at the different experimental conditions (liquid phase composition and gas flow-rate) assayed in this work (Figure 5).

Experimental results shown in Figure 5 indicate the influence of liquid phase composition and gas flow-rate upon the specific interfacial area generated in the bubble column. The increase of the gas flow-rate leads to an increase of the interfacial area also increases due to the previously observed behaviour caused by this variable upon the gas hold-up. The gas hold-up increases with the gas flow-rate due to a higher number of bubbles being generated in the sparger, increasing the interfacial area.

When pure water was employed as liquid phase in the bubble contactor at high values of gas flow-rate, that increase was not observed because under those conditions there is a great number of bubbles that collide producing coalescence and therefore increasing the bubbles diameter. 


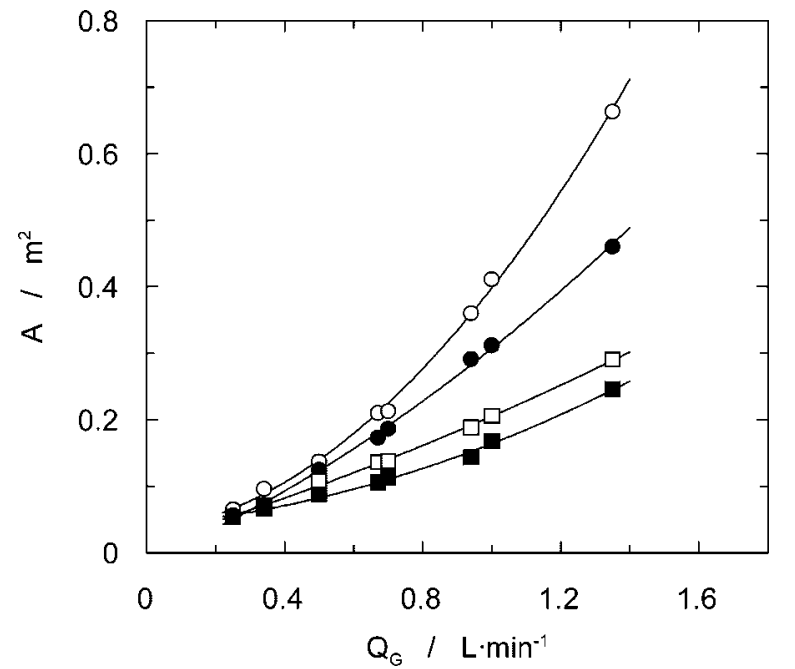

Figure 5. Influence of gas flow-rate and methyl ricinoleate concentration on the interfacial area in the airlift bioreactor. All systems have $0.093 \%$ (v/v) Tween-80 in their composition. ( ) $0 \%(\mathrm{v} / \mathrm{v}) \mathrm{MR}$; (•) $0.1 \%(\mathrm{v} / \mathrm{v}) \mathrm{MR}$;

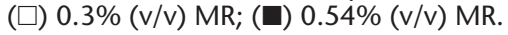

The presence of surfactant in the liquid phase produces a clear increase in the specific interfacial area value compared to the corresponding value for pure water. That increase is more significant when the gas flow-rate increases. This behaviour is due to different reasons. One of them is the clear decrease in the liquid phase surface tension when the surfactant concentration increases (Gómez-Díaz et al., 2008) as it causes the formation of bubbles with minor size increasing the interfacial area. Furthermore, the presence of surfactant molecules in the liquid phase produces a decrease in the bubbles effective collisions and therefore the bubbles small size remains constant along the bubble column.

The experimental results obtained when emulsions were employed as liquid phases are presented in Figure 5, indicating a decrease in the specific interfacial area value when the oil concentration increases in the liquid phase. This effect is negligible for the lowest gas flow-rate analysed but when this variable increases, the presence of the organic phase has an important role on the values of interfacial area. This behaviour is assigned to a change in the surfactant concentration at the gas-liquid interface because the surface and interfacial tensions determined for this experimental system indicate that when an organic phase is added to aqueous solutions with Tween-80, a new distribution of surfactant between interface and bulk phases is originated. The experimental results indicate that an important decrease in the surfactant concentration in the gas-liquid interface is produced because the value of surface tension increases. Also, the value of interfacial tension decreases, due to an increase in the surfactant concentration at the liquid-liquid interface. This distribution affects the liquid phase surface tension value and consequently the bubbles produced under these conditions have higher diameters than those formed in the absence of the organic phase.

\section{NOMENCLATURE}

$\begin{array}{ll}a & \text { specific interfacial area }\left(\mathrm{m}^{-1}\right) \\ A & \text { interfacial area }\left(\mathrm{m}^{2}\right) \\ d & \text { equivalent bubble diameter }(\mathrm{m}) \\ d_{32} & \text { Sauter mean diameter }(\mathrm{m}) \\ e & \text { Minor ellipsoide axe }(\mathrm{m}) \\ E & \text { Major ellipsoide axe }(\mathrm{m})\end{array}$

$n \quad$ number of bubbles $(-)$

$Q_{\mathrm{G}} \quad$ gas flow-rate $\left(\mathrm{m}^{3} \mathrm{~s}^{-1}\right)$

$U_{\mathrm{L}} \quad$ superficial liquid velocity $\left(\mathrm{m} \mathrm{s}^{-1}\right)$

$V \quad$ total volume $\left(\mathrm{m}^{3}\right)$

$V_{\mathrm{L}} \quad$ liquid volume $\left(\mathrm{m}^{3}\right)$

\section{Greek Symbols}

$\varepsilon_{\mathrm{G}} \quad$ gas hold-up

\section{REFERENCES}

Aguedo, M., N. Gomes, E. Escamilla-Garcia, Y. Waché, M. Mota, J. A. Teixeira and I. Belo, "Decalactone Production by Yarrowia lipolytica Under Increased $\mathrm{O}_{2}$ Transfer Rates,” Biotech. Lett. 27, 1617-1621 (2005).

Dumont, E. and H. Delmas, "Mass Transfer Enhancement of Gas Absorption in Oil-in-Water Systems: A Review," Chem. Eng. Process 42, 419-438 (2003).

Gomes, N., M. Aguedo, J. A. Teixeira and I. Belo, “Oxygen Mass Transfer in a Biphasic Medium: Influence on the Biotransformation of Methyl Ricinoleate Into $\gamma$-Decalactone by the Yeast Yarrowia lipolytica," Biochem. Eng. J. 35, 380-386 (2007).

Gómez-Díaz, D., J. M. Navaza and B. Sanjurjo, "Interfacial Area Evaluation in a Bubble Column in the Presence of a Surface-Active Substance: Comparison of Methods," Chem. Eng. J. 144, 379-385 (2008).

Shah, Y. T., B. G. Kelkar, S. P. Godbole and W. D. Deckwer, "Design Parameters Estimation for Bubble Column Reactors," AIChE J. 28, 353-379 (1982).

van't Riet, V. and J. Tramper, “Basic Bioreactor Design,” Marcel Dekker, New York, USA (1991).

Vasconcelos, J. M. T., J. M. I. Rodrigues, S. C. P. Orvalho, S. S. Alves, R. L. Mendes and A. Reis, "Effect of Contaminants on Mass Transfer Coefficients in Bubble Column and Airlift Contactors,” Chem. Eng. Sci. 58, 1431-1440 (2003).

Manuscript received November 19, 2009; revised manuscript received January 7, 2010; accepted for publication January 7, 2010 